\title{
Dictynna
}

Dictynna

Revue de poétique latine

17 | 2020

International Ovidian Society en Europe I

\section{Le imprese degli eroi: strutture catalogiche nell'Eneide e nelle Metamorfosi}

\section{Mario Labate}

\section{(2) OpenEdition}

Journals

\section{Edizione digitale}

URL: https://journals.openedition.org/dictynna/2317

DOI: $10.4000 /$ dictynna.2317

ISSN: 1765-3142

\section{Editore}

Université de Lille

\section{Notizia bibliografica digitale}

Mario Labate, "Le imprese degli eroi: strutture catalogiche nell'Eneide e nelle Metamorfosi », Dictynna [En ligne], 17 | 2020, mis en ligne le 17 décembre 2020, consulté le 15 décembre 2022. URL : http:// journals.openedition.org/dictynna/2317 ; DOI : https://doi.org/10.4000/dictynna.2317

Questo documento è stato generato automaticamente il 15 décembre 2022.

\section{(c) (i) (9)}

Creative Commons - Attribution - Pas d'Utilisation Commerciale - Pas de Modification 4.0 International - CC BY-NC-ND 4.0

https://creativecommons.org/licenses/by-nc-nd/4.0/ 


\title{
Le imprese degli eroi: strutture catalogiche nell'Eneide e nelle Metamorfosi
}

\author{
Mario Labate
}

1 L'epica omerico-virgiliana aveva concesso uno spazio molto limitato a quelle zone dell'immaginario che si dispongono attorno a figure di eroi diversi da Achille ed Ettore, da Enea e Turno: eroi più antichi e fantastici, che misurano il proprio valore soprattutto come liberatori da mostri e flagelli che opprimono l'umanità. Questi eroi 'più grandi' si muovono quasi sempre in solitudine e combattono contro avversari terrificanti, se non addirittura con la sola forza delle mani, con armi primitive, la cui efficienza dipende dalle straordinarie doti di chi le impugna. Sappiamo che questi eroi avevano alimentato un'ampia produzione di Eracleidi e di Teseidi, vitale dall'età arcaica all'età ellenistica. In particolare la poesia ellenistica, non soltanto la poesia epica ma anche l'epos in miniatura, l'elegia, l'epigramma erano fortemente attratti da queste zone del mito tradizionalmente frequentate dai poeti del ciclo, dalla lirica, dalla tragedia ${ }^{1}$.

2 La figura di Ercole (in parte anche quella di Teseo) funziona come una specie di catalizzatore dell'immaginario epico fantastico-iperbolico, ma, nella tradizione ellenistico-romana, le gesta di questi eroi più grandi non erano per lo più destinate ad occupare da protagoniste la narrazione di un grande poema epico (come nelle Eracleidi e nelle Teseidi condannate da Aristotele), ma piuttosto ad essere chiamate in scena episodicamente o addirittura marginalmente: si potrebbe anzi supporre che proprio le obiezioni di Aristotele relative alla mancanza di vera unitarietà di quei poemi il cui progetto narrativo comprendeva tutte le diverse storie relative ad un unico personaggio, ${ }^{2}$ come Ercole o Teseo, segnalasse di fatto il ricchissimo materiale mitico relativo a questi eroi come particolarmente indicato per una utilizzazione episodica in narrazioni epiche di compasso ridotto o attraverso una presenza obliqua in storie di carattere diverso.

3 La complessiva vicenda mitica di questi eroi, archiviata come materia del grande poema epico, può tuttavia trovare spazio in succinte rievocazioni catalogiche, che mutuano 
moduli della poesia lirica o drammatica e che hanno soprattutto la funzione di evocare un mondo suggestivo e remoto, che al tempo stesso è indispensabile come quadro di riferimento, ma che è anche ingombrante e la cui gestione diretta da parte di un poeta moderno risulterebbe non poco problematica.

4 Secondo un epigramma di Teocrito che costituisce una importante testimonianza per gli Herakleia di Pisandro, l'opera di quest'ultimo parlava di "tutte le fatiche che sopportò":

Theocr. epigr. $22(A P$ 9, 598)

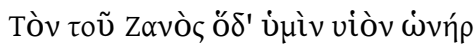

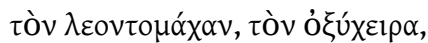

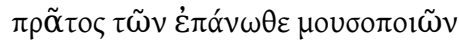

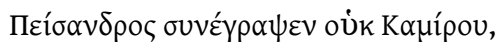

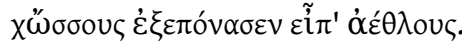

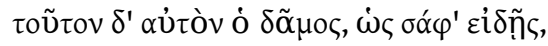

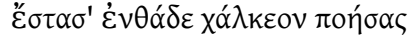

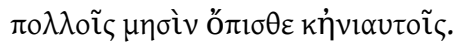

“Del figlio di Zeus che combatté con il leone, dell'eroe pronto di mano, e di tutte le imprese che compì, primo tra i poeti del passato per voi scrisse Pisandro di Camiro.

Il popolo, sappilo bene, una statua di bronzo qui gli eresse, a distanza di molti mesi

e anni" (trad. B. M. Palumbo Stracca).

Quanti e quali fossero gli ǒ $\theta \lambda$ or di cui scrisse Pisandro non è dato sapere con esattezza, e niente si sa di come la lista delle fatiche fosse strutturata negli Herakleia di Paniassi. Delle serie consistenti, variabili per numero e ordine, ci sono testimoniate in Sofocle ed Euripide e varie testimonianze letterarie e figurative ci fanno pensare a una progressiva fissazione, nel $\mathrm{V}$ secolo, di un elenco canonico di imprese che ne identificava dodici come quelle compiute al servizio di Euristeo, cui se aggiungevano numerose altre variamente collocate nella cronologia relativa del mito. In età ellenistica, le "dodici fatiche di Ercole" sono indicate come un 'insieme' condiviso, anche se non abbiamo testimonianze, se non parziali, di quali fossero quelle che costituivano l'elenco. Si veda ad esempio la profezia di Tiresia ad Alcmena nell'Herakliskos di Teocrito:

Theocr. 24, 79-85

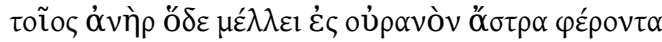

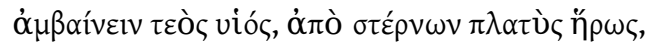

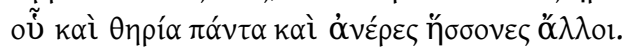

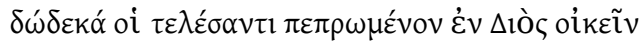

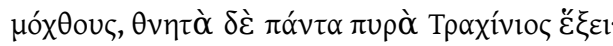

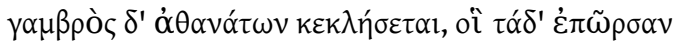

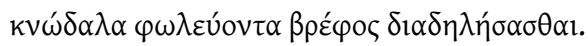

“Tal uomo questo tuo figlio al cielo stellato ascenderà, eroe dall'ampio petto, degli uomini tutti e delle fiere assai più forte. Dopo aver compiuto dodici fatiche, è destinato che abbia dimora nella casa di Zeus, e la pira trachinia avrà le sue spoglie mortali. Sarà chiamato genero degli stessi immortali che questi mostri, abitatori di caverne, suscitarono, a distruggerlo bambino." (trad. B. M. Palumbo Stracca)

La serie canonica si stabilizza, con differenze ormai lievi, solo in fonti, soprattutto mitografiche, di età tarda (Diodoro 4, 8-27; [Apollod.] 2, 74-126; Hyg. Fab. 30; Quint. Smyrn. 6, 200-68; Serv. ad Aen. 8, 299 (incompleto) . $^{3}$.

7 Non sono tuttavia le vicende che hanno portato alla costituzione e alla fissazione di questo canone che ci interessano qui, quanto piuttosto il fatto stesso che la serie esista come tale e che tenda a condizionare in maniera significativa la rappresentazione 
letteraria dei singoli episodi mitici, che, pur dotati di una autonomia narrativa e simbolica, sono tuttavia sempre condizionati dalla loro appartenenza a una serie ${ }^{4}$. Gli eroi come Ercole (e Teseo) sono cioè protagonisti di singole storie che sono in sé concluse, ma che al tempo stesso condividono un marchio di serialità, rimandano le une alle altre, a quelle passate e a quelle future nella cronologia del mito, perché da tutte assieme risulti quella fisionomia di eroe liberatore e benefattore dell'umanità che diviene il prototipo di ogni personalità eccezionale destinata, dopo un percorso cominciato nella culla e finito sulla pira funebre, all'assunzione in cielo fra gli dèi.

Che la vicenda degli eroi dell'epica possa essere costituita da una pluralità di episodi è naturalmente una caratteristica che riguarda più in generale il poema di viaggio e di avventura. Anche Ulisse misurava le proprie qualità eroiche contro una serie di avversari e di situazioni insidiose o pericolose, che la tradizione letteraria e mitografica successiva potrà riproporre in un quadro sinottico. Ma il caso di Ulisse, come del resto quello di Giasone e degli Argonauti, o anche quello di Enea, sono diversi, in tanto in quanto i singoli episodi si collocano in una vicenda articolata concepita come 'unitaria': il nostos di Ulisse, il viaggio degli Argonauti, il viaggio / nostos di Enea. Dal punto di vista formale la serie degli episodi può essere rappresentata come un più o meno succinto riassunto dell'opera. Per restare al caso dell'Odissea, a livello di fonti mitografiche disponiamo di circostanziati resoconti, nello pseudo-Apollodoro e in Igino; quest'ultimo, ad esempio, in ben 874 parole snocciola, seguendo abbastanza fedelmente la falsariga del poema (Vlixes cum ab Ilio in patriam Ithacam rediret...), i seguenti episodi: 1) Ciconi 2) Lotofagi 3) Polifemo 4) Eolo 5) Lestrigoni 6) Circe 7) Nekyia 8) Sirene 9) Scilla 10) Vacche del Sole 11) Cariddi 12) Calipso 13) Feaci 14) Proci.

9 L'abregé poetico è naturalmente assai più succinto, incompleto, nonché più orientato alle esigenze del contesto letterario in cui si trova ad essere inserito, anche se mantiene pur sempre la struttura narrativa dell'originale. Si veda ad esempio l'elegia 3, 12 di Properzio, in cui le avventure di Ulisse sono ripercorse rapidamente allo scopo di operare una synkrisis tra la incrollabile fedeltà di Penelope al suo sposo pur sottoposto a tante traversie, e quella, non meno esemplare, di Galla, la sposa di Postumo, cui l'elegia è indirizzata ${ }^{5}$ :

Prop. 3, 12, 23-38

Postumus alter erit miranda coniuge Ulixes: non illi longae tot nocuere morae,

castra decem annorum, et Ciconum mors, Ismara capta, exustaeque tuae nox, Polypheme, genae,

et Circae fraudes, lotosque herbaeque tenaces, Scyllaque et alternas scissa Charybdis aquas,

Lampeties Ithacis veribus mugisse iuvencos (paverat hos Phoebo filia Lampetie),

et thalamum Aeaeae flentis fugisse puellae, totque hiemis noctes totque natasse dies, nigrantisque domos animarum intrasse silentum, Sirenum surdo remige adisse lacus,

et veteres arcus leto renovasse procorum, errorisque sui sic statuisse modum.

nec frustra, quia casta domi persederat uxor. vincit Penelopes Aelia Galla fidem.

"Postumo grazie alla sua ammirevole sposa sarà un secondo Ulisse: a lui non nocquero tante e così lunghe assenze, l'assedio di dieci anni e la strage dei Ciconi, la presa di Ismaro, e poi le tue orbite bruciate, Polifemo, l'inganno di Circe, e il loto e 
le erbe che trattengono, e Scilla, e Cariddi che nel risucchio in due acque si scinde, né il muggito dei buoi di Lampezia sugli spiedi itacesi (per Febo li pasceva sua figlia Lampezia), né la fuga dal letto della fanciulla eea in lacrime, e le tante notti e i tanti giorni nel mare in tempesta, e la discesa alle oscure dimore delle anime silenti ed avere accostato con i sordi rematori le acque delle Sirene, e aver ripreso in mano il vecchio arco per la strage dei proci, ponendo così termine alle sue peregrinazioni. Non invano, perché la sposa aveva mantenuto la sua castità nella casa. La fedeltà di Penelope Elia Galla la supera."

L'accentuazione del carattere tendenzioso del racconto tende tuttavia a fare dei singoli episodi degli exempla staccati, assemblabili secondo una logica propria e in un ordine diverso da quello 'di provenienza'. Un più chiaro esempio di riassunto 'tendenzioso' è nelle elegie dell'esilio di Ovidio dove il resoconto dei labores di Ulisse è costantemente indirizzato a minimizzarne la portata nella synkrisis con il poeta stesso, vero campione di dolorosa patientia ${ }^{6}$ :

Ov. ex P. 4, 10, 9-30

Exemplum est animi nimium patientis Vlixes iactatus dubio per duo lustra mari,

tempora solliciti sed non tamen omnia fati pertulit et placidae saepe fuere morae.

An graue sex annis pulchram fouisse Calypson aequoreaeque fuit concubuisse deae?

Excipit Hippotades qui dat pro munere uentos, curuet ut inpulsos utilis aura sinus.

Nec bene cantantis labor est audire puellas nec degustanti lotos amara fuit.

Hos ego qui patriae faciant obliuia sucos parte meae uitae, si modo dentur, emam.

Nec tu contuleris urbem Laestrygonos umquam gentibus obliqua quas obit Hister aqua, nec uincet Cyclops saeuum feritate Piacchen; qui quota terroris pars solet esse mei?

Scylla feris trunco quod latret ab inguine monstris, Heniochae nautis plus nocuere rates.

Nec potes infestis conferre Charybdin Achaeis, ter licet epotum ter uomat illa fretum;

qui quamquam dextra regione licentius errant, securum latus hoc non tamen esse sinunt.

"Modello di animo oltremodo paziente è Ulisse, sbattuto per due lustri sul mare infido; tuttavia non subì in ogni momento un destino angosciante, e vi furono spesso intervalli tranquilli. Forse è stato gravoso stringere tra le braccia la bella Calipso per sei anni e giacere con la dea marina? Lo accoglie l'Ippotade, che in dono gli dà i venti, affinché un soffio favorevole incurvi e spinga le sue vele; non è una fatica udire fanciulle dal canto soave, e il loto non fu amaro al gusto: questo succo che produce l'oblio della patria io lo comprerei, se solo mi venisse dato, con una parte della mia vita. E tu non potresti mai paragonare la città dei Lestrigoni con le genti che l'Istro tocca con le sue anse: né il Ciclope vincerà in ferocia il crudele Piacche, che del resto è solo una parte del mio terrore. E quanto a Scilla che latra dall'inguine tronco con i mostri selvaggi: le navi degli Eniochi hanno fatto più danni ai naviganti. E con Cariddi, benché vomiti tre volte il mare che tre volte ha bevuto, non puoi porre a confronto gli ostili Achei, che anche se è nella regione orientale che si muovono più liberamente, non consentono tuttavia che questa sponda sia senza pericolo." (trad. L. Galasso)

11 La forma del riassunto di narrazioni epiche 'aristotelicamente' unitarie è suscettibile di essere inglobata in strutture narrative più ampie. Nelle Metamorfosi Ovidio, come è ben 
noto, sperimenta in maniera originale questa possibilità nella 'piccola Eneide' dei libri 13-14 in cui un sintetico abregé virgiliano del viaggio di Enea costituisce un canovaccio in cui inserire opportunamente storie di metamorfosi ${ }^{7}$.

Per quanto riguarda invece gli eroi più grandi, la poesia augustea, sia con Virgilio che con Ovidio, sceglie una strada diversa: quella della narrazione ampia di singoli episodi in cui si dispiegano le qualità eroiche del personaggio (Ercole contro Caco in Virgilio, Ercole contro Acheloo, Ercole contro Nesso in Ovidio), ma al tempo stesso senza rinunciare alla possibilità, anzi quasi alla necessità, di un riferimento trasversale ad altre storie della serie e anzi alla serie nel suo complesso.

Nella lotta tra Ercole e Acheloo, ${ }^{8}$ sono anzitutto le capacità metamorfiche del dio fluviale, oltre a motivare l'accoglimento di questa impresa fra quelle che hanno particolare diritto di cittadinanza nel poema, a dare occasione di un ironico intreccio con le fatiche, canoniche e no ${ }^{10}$. Nel duello atletico ${ }^{11}$, impotente di fronte alla forza preponderante dell'avversario, Acheloo ricorre alle sue artes trasformandosi in serpente per sgusciare dalla presa e minacciare l'eroe nella nuova forma. E' appunto la serie delle imprese che permette ad Ercole di mettere in ridicolo questa presunta minaccia, estraendo da essa una sub-categoria specifica "Ercole contro i serpenti", che comincia con la sua prima performance di bambino, che con irrisoria facilità strozza $i$ draghi inviati da Era e culmina con la spettacolare impresa dell'Idra di Lerna, che Ercole stesso rievoca con virtuosistica abilità narrativa nella descrizione del prodigioso rigenerarsi del mostro, ma con singolare reticenza sul modo in cui egli ne aveva avuto ragione (con il determinante aiuto di Iolao). Il poliptoto costituito da un verbo di forma finita ripreso da un participio ostenta appunto una facilità estrema dell'operazione di fronte all'estrema difficoltà dell'impresa (domui domitamque reclusi):

Ov. met. 9, 62-76

inferior virtute, meas devertor ad artes, elaborque viro longum formatus in anguem. qui postquam flexos sinuavi corpus in orbes, cumque fero movi linguam stridore bisulcam, risit, et inludens nostras Tirynthius artes "cunarum labor est angues superare mearum," dixit "et ut vincas alios, Acheloe, dracones, pars quota Lernaeae serpens eris unus echidnae? vulneribus fecunda suis erat illa, nec ullum de centum numero caput est inpune recisum, quin gemino cervix herede valentior esset. hanc ego ramosam natis e caede colubris crescentemque malo domui, domitamque reclusi. quid fore te credis, falsum qui versus in anguem arma aliena moves, quem forma precaria celat?"

"Inferiore di forze, ricorro alle mie abilità e sfuggo al rivale in forma di lungo serpente. Vedendomi spiegare il mio corpo in spire sinuose, e vibrare la bifida lingua in sibili agghiaccianti, l'eroe tirinzio sbottò a ridere e, dileggiando i miei trucchi, 'Fin dalla culla mi diverto a sconfiggere i serpenti' disse 'e se anche, Acheloo, tu fossi il più forte dei draghi, cosa mai saresti, tu solo, a cospetto del mostro di Lerna? Dalle sue stesse ferite colei si rigenerava e nessuna delle sue teste poteva essere recisa, senza che, dal collo, ne rispuntassero due più sane di prima. $\mathrm{E}$ io un mostro così, che le teste mozzate diramava in nuovi serpenti rinascendo da ogni sua piaga, l'ho domato, poi anche squartato. E tu cosa credi di fare, mutato in falso serpente, sfoggiando armi non tue e celato in una forma d'accatto?" (trad. di G. Chiarini, con ritocchi) 
Un riferimento trasversale implicito a un'altra impresa della serie è probabilmente da ravvisare nel successivo momento della lotta di Ercole con Acheloo, quando il dio fluviale ricorre alla sua estrema risorsa, la trasformazione in toro:

Ov. met. 9, 80-6

sic quoque devicto restabat tertia tauri

forma trucis. tauro mutatus membra rebello.

induit ille toris a laeva parte lacertos,

admissumque trahens sequitur, depressaque dura

cornua figit humo, meque alta sternit harena.

nec satis hoc fuerat: rigidum fera dextera cornu

dum tenet, infregit, truncaque a fronte revellit.

"Vinto anche in questa forma, non mi restava che quella selvaggia di un toro: mutatomi in toro, riprendo la lotta. Lui dal fianco sinistro mi abbranca la giogaia, asseconda il mio slancio e mi trascina, mi piega ficcandomi le corna nella dura terra e m'abbatte in un gran polverone. Non basta: mentre m'afferra un rigido corno con mano feroce, me lo spezza strappandolo alla fronte amputata." (trad. di G. Chiarini)

E' verisimile, a questo punto, presupporre un lettore di Ovidio in grado di creare da sé una sub-categoria "Ercole contro il toro", che richiamasse quella che, nel computo tradizionale, era considerata come la settima fatica, il toro di Creta: quella, fra l'altro, che più direttamente di altre creava una sovrapposizione tra i due eroi liberatori dai mostri, se è vero che la più nota o per lo meno la più letterariamente prestigiosa delle imprese di Teseo (narratario della storia raccontata dallo sconfitto Acheloo ${ }^{12}$ ), è appunto il toro di Maratona, che era considerato lo stesso mostruoso animale precedentemente catturato da Ercole a Creta ${ }^{13}$.

Nell'Eneide l'impresa di Ercole contro Caco (una leggenda italica assai meno consolidata nella tradizione mitografica e a cui Virgilio apporta tra l'altro significative varianti) è immaginata come una appendice della decima fatica, l'uccisione, all'estremo occidente del mondo, del mostruoso Gerione e la cattura del suo bestiame ${ }^{14}$. Il corpo a corpo poderoso con cui Ercole riesce a ridurre all'impotenza e a uccidere il suo infernale avversario Caco incrocia per tratti significativi lo scontro con Anteo, con Acheloo, con il leone nemeo stesso. Più ancora conta il fatto che, a conclusione del racconto di Evandro, è la serie nel suo complesso che viene evocata come quella cui ora l'uccisione di Caco si aggiunge come ulteriore fatica. La festa annuale all'Ara Massima era l'occasione per il racconto eziologico di Evandro. A conclusione del racconto, sempre i rituali in onore di Ercole prevedono l'esecuzione da parte dei Salii di un inno all'eroe ${ }^{15}$ che contiene un catalogo delle sue gesta, che ha soprattutto la funzione di accreditare come 'degna di Ercole', eroe dell'autocontrollo e della forza razionale, quella ulteriore impresa, in cui in effetti l'eroe aveva rischiato di assomigliare anche troppo al suo furibondo e animalesco avversario ${ }^{16}$ :

Verg. Aen. 8, 285-304

tum Salii ad cantus incensa altaria circum populeis adsunt euincti tempora ramis, hic iuuenum chorus, ille senum, qui carmine laudes Herculeas et facta ferunt: ut prima nouercae monstra manu geminosque premens eliserit anguis, ut bello egregias idem disiecerit urbes, Troiamque Oechaliamque, ut duros mille labores rege sub Eurystheo fatis Iunonis iniquae pertulerit. 'tu nubigenas, inuicte, bimembris Hylaeumque Pholumque manu, tu Cresia mactas prodigia et uastum Nemeae sub rupe leonem. 
te Stygii tremuere lacus, te ianitor Orci

ossa super recubans antro semesa cruento;

nec te ullae facies, non terruit ipse Typhoeus

arduus arma tenens; non te rationis egentem

Lernaeus turba capitum circumstetit anguis.

salue, uera Iouis proles, decus addite diuis,

et nos et tua dexter adi pede sacra secundo.'

talia carminibus celebrant; super omnia Caci

speluncam adiciunt spirantemque ignibus ipsum.

"Allora i Salii intorno agli altari accesi giungono al canto con le tempie avvinte di fronde di pioppo; questo il coro dei giovani, quello dei vecchi; narrano nel carme le glorie e le imprese di Ercole; come, serrando la mano, strozzò per primi i mostri della matrigna, i due serpenti; e in guerra distrusse famose città, e Troia ed Ecalia, e sopportò mille dure fatiche sotto il re Euristeo, per volere dell'iniqua Giunone. 'Tu, o invitto, immoli di tua mano i figli della nube dalle duplici membra, Ileo e Folo, e il mostro cretese e il grande leone sotto la rupe nemea. Per te tremarono i laghi stigii, e il guardiano dell'Orco disteso su ossa semidivorate nell'antro sanguinoso. Non ti atterrì nessun mostro, neanche Tifeo che alto levava le armi; né l'Idra di Lerna ti privò del senno circondandoti con la turba di teste. Salve, vera progenie di Giove, nuova gloria degli dei, visita noi e il tuo sacrificio con passo propizio.' Questo celebrano nei carmi; e soprattutto aggiungono la spelonca di Caco, e lui che spira fiamme." (trad. L. Canali)

17 Virgilio rende così esplicito quale sia il modello formale che, anche all'interno della narrazione epica, può e deve assumere il riferimento di tipo catalogico alla serie delle imprese di eroi che non possono essere considerati protagonisti di una trama epica unitaria secondo i dettami di Aristotele: la forma è quella dell'inno e in particolare

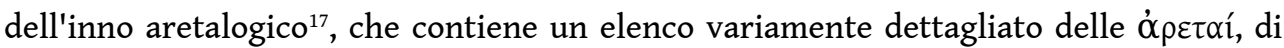
una divinità. Il catalogo degli ó $\theta \lambda$ or di Ercole del Carmen saliare virgiliano è contenuto in un canto corale che ha per tema laudes Herculeas et facta, "le gesta gloriose di Ercole", riferite prima in forma indiretta (ut...ut...ut...) , poi con un inno al dio nel Du-stil e con le altre caratteristiche formali della preghiera.

18 La matrice lirico-tragica di questo modulo è confermata dall'emulazione di Ovidio, che nelle Metamorfosi la trasferisce a Teseo, l'eroe tipologicamente affine e a sua volta emulo di Ercole. Giunto a destinazione dopo il viaggio da Trezene ad Atene che costituiva il contesto della maggior parte delle sue imprese di eroe liberatore, Teseo, grazie alla provvidenziale $\dot{\alpha} v \alpha \gamma v \omega ́ p ı \sigma ı$, , sfugge in extremis alla morte che, per le trame di Medea, il suo stesso inconsapevole padre Egeo sta per infliggergli. Allo scampato pericolo seguono festeggiamenti e celebrazioni dell'eroe, finalmente riconosciuto non come un pericolo, ma come il liberatore dai mostri e dai flagelli che infestano la regione.

19 Nel Ditirambo 4 di Bacchilide un resoconto delle imprese di Teseo nell'Istmo era contenuto nella seconda strofe del dialogo lirico in cui Egeo risponde al coro degli Ateniesi, preoccupati dallo squillo di tromba che è appena risuonato, possibile segnale di pericolo ${ }^{18}$. Egeo riferisce quanto ha a sua volta riferito un araldo, che aveva narrato "le indescrivibili imprese di un uomo possente": l'uccisione 1) di Sini, 2) della scrofa di Cremmione (Crommione), 3) di Scirone 4) di Cercìne 5) di Procopte (Procuste). La serie sostanzialmente corrisponde a quelle della vita plutarchea e delle fonti mitografiche (ps-Apollodoro, Igino). In Bacchilide, la serie rispetta la struttura narrativa del resoconto dell'araldo: Egeo, del resto, esprime ancora incertezza e timore sul possibile sito della vicenda (un nuovo pericolo o la salvezza dal pericolo?): 
Bacchil. Dithyr. 4, 16-30

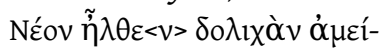

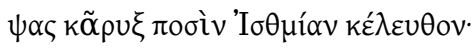

ö $\varphi \alpha \tau \alpha \delta^{\prime} \varepsilon^{\prime} \rho \gamma \alpha \lambda \varepsilon^{\prime} \gamma \varepsilon l \kappa \rho \alpha \tau \alpha l^{-}$

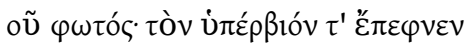

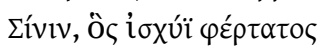

$\theta v \alpha \tau \tilde{\omega} v \tilde{\tilde{\eta}} \nu, K \rho o v i ́ \delta \alpha \Lambda v \tau \alpha i ́-$

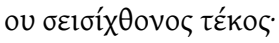

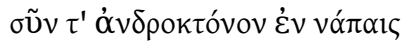

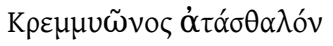

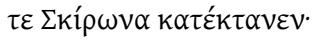

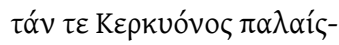

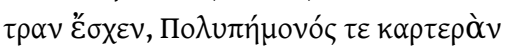

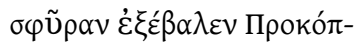

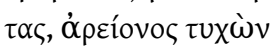

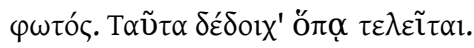

"E' appena giunto un araldo

che ha traversato a piedi il lungo cammino dell'Istmo.

Narra delle indescrivibili imprese di un uomo

possente. Ha abbattuto il figlio del Cronide

Liteo scuotiterra, il violento

Sini, che per vigore era più forte

di ogni mortale.

Ha ucciso la scrofa assassina

nelle valli di Cremmione

così come il superbo Scirone.

Si è impadronito della palestra

di Cercione, e Procopte, imbattutosi

in qualcuno più forte di lui,

ha lasciato cadere il possente maglio di Polipemone.

Ho timore di come ciò possa andare a finire." (trad. Giuseppetti, con ritocchi)

Non sappiamo come e quanto ampiamente le imprese di Teseo fossero riferite nel poema (una Teseide?) con cui Albinovano Pedone aveva forse osato la sfida del grande poema sulle imprese degli eroi più grandi ${ }^{19}$, ma non è escluso che il poema ospitasse moduli della tradizione innica: (Ov. Ex P. 4, 10, 71 cum Thesea carmine laudes), Ovidio esule richiama comunque l'amico poeta a una emulazione meno eroica del suo protagonista, alla emulazione dell'eroe della fides, più che del domatore dei mostri:

Ov. ex P. 4, 10, 75-82

Qui quamquam est factis ingens et conditur a te uir tanto quanto debuit ore cani,

est tamen ex illo nobis imitabile quiddam inque fide Theseus quilibet esse potest.

Non tibi sunt hostes ferro clauaque domandi, per quos uix ulli peruius Isthmos erat,

sed praestandus amor, res non operosa uolenti. Quis labor est puram non temerasse fidem?

"Ma ecco, tu celebri Teseo nel tuo carme e non dubito che tu ti poni alla pari dei titoli di gloria del tuo argomento e che imiti l'eroe di cui racconti: di certo egli vieta che la Lealtà sia la compagna solo dei tempi tranquilli. E benché quell'eroe sia grande per le sue imprese e sia da te celebrato con la voce tanto sonora che gli è dovuta, anche per noi c'è in lui qualcosa che si può imitare e nella lealtà chiunque può essere un Teseo. Non hai da sottomettere con la spada e con la clava i nemici, a causa dei quali l'Istmo non era percorribile praticamente per nessuno, ma bisogna 
dimostrare amicizia, un compito non impegnativo per chi sia ben disposto: che fatica è non insozzare una lealtà immacolata?" (trad. L. Galasso)

21 Nelle Metamorfosi (come nell'Eneide per Ercole), dopo il riconoscimento che ha dissipato le inquietudini di Egeo, si celebra la gratitudine al liberatore e il sollievo per lo scampato nefas nella forma solenne dell'inno in Du-Stil ${ }^{20}$, la più opportuna a presentare il catalogo degli ơ $\theta \lambda$ or dell'eroe, che comprende, oltre a quelli enumerati in Stesicoro, il più recente (il toro di Maratona ) e il primo, l'uccisione a Epidauro di Perifete:

Ov. met. 7, 430-50

nullus Erecthidis fertur celebratior illo

inluxisse dies: agitant convivia patres

et medium vulgus nec non et carmina vino

ingenium faciente canunt: 'te, maxime Theseu,

mirata est Marathon Cretaei sanguine tauri,

quodque suis securus arat Cromyona colonus,

munus opusque tuum est; tellus Epidauria per te

clavigeram vidit Vulcani occumbere prolem,

vidit et inmitem Cephisias ora Procrusten,

Cercyonis letum vidit Cerealis Eleusin.

occidit ille Sinis magnis male viribus usus, qui poterat curvare trabes et agebat ab alto ad terram late sparsuras corpora pinus.

tutus ad Alcathoen, Lelegeia moenia, limes

conposito Scirone patet, sparsisque latronis

terra negat sedem, sedem negat ossibus unda;

quae iactata diu fertur durasse vetustas

in scopulos: scopulis nomen Scironis inhaeret.

si titulos annosque tuos numerare velimus,

facta prement annos. pro te, fortissime, vota

publica suscipimus, Bacchi tibi sumimus haustus.'

"Nessun giorno di festa si dice splendesse più radioso per la stirpe di Eretteo. Banchettano i nobili con la gente comune, e intonano pure canti ispirati dal vino: 'Te, sommo Teseo, Maratona ha ammirato per il sangue versato dal toro cretese, e se a Crommione il contadino ara senza temere la scrofa, ciò è opera e merito tuo. La terra d'Epidauro grazie a te ha visto soccombere il figlio armato di clava di Vulcano, e la valle del Cefiso ha visto cadere il crudele Procuste, ed Eleusi di Cerere ha visto la morte di Cercione. Morto è pure Sini, che male usava la sua grande forza, che era capace di piegare i tronchi e piegava la cima di un pino fino a terra per catapultare i corpi lacerati tutt'intorno. La via per Alcatoe, rocca dei Lelegi, s'apre sicura, sistemato Scirone, e alle ossa sparse del bandito nega una sede la terra, nega una sede l'onda del mare, e dicono che a lungo sbattute si indurissero in antichi scogli: a tali scogli è rimasto il nome di Scirone. A elencare i tuoi meriti e i tuoi anni, le imprese supererebbero gli anni! Per te, o fortissimo, facciamo pubblici voti, alla tua salute sorseggiamo questo vino'." (trad. G. Chiarini)

Nella celebrazione delle imprese degli eroi più grandi, questi moduli lirici avevano un punto di snodo fondamentale nel primo stasimo dell'Eracle di Euripide. Caratteristica di questo celebre canto corale dedicato alle fatiche dell'eroe è quella di essere in bilico tra due possibili funzioni, quella di celebrazione / glorificazione e quella di lamentazione funebre ${ }^{21}$. Come Anfitrione annunciava nel prologo, Eracle "ha portato a termine le altre fatiche, ma per l'ultima è andato nell'Ade attraverso la bocca del Tenaro, per condurre alla luce il cane dal triplice corpo, e non ne è più tornato" (Eur. Her. 22-5). Da questa situazione drammatica scaturisce la commistione di moduli innici e moduli trenodici che l'esordio del coro programmaticamente annuncia e a cui seguirà l'ampio catalogo di 12 imprese (non tutte comprese in quello che sarà l'elenco canonico): 
Eur. Her. 348-59

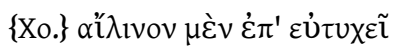

$\mu \circ \lambda \pi \tilde{\alpha}_{1}$ Фог̃ßos i $\alpha \chi \varepsilon \tilde{\imath}$

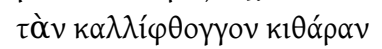

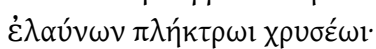

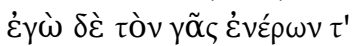

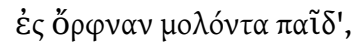

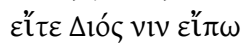

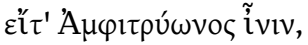

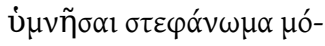

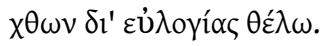

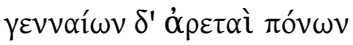

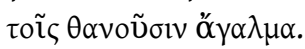

"Ailinos è il grido luttuoso

che Febo intona dopo un canto di trionfo,

toccando la cetra dal suono armonioso

con l'aureo plettro;

e io per l'eroe che è sceso nelle tenebre della terra, agli Inferi,

sia che lo si debba chiamare rampollo di Zeus

o di Anfitrione,

voglio intonare un inno, corona intrecciata

con l'encomio delle sue fatiche.

La gloria delle imprese nobili

è ornamento per i morti." (trad. M. S. Mirto)

Approfittando di questa duplice possibilità, Ovidio rinuncia, per le imprese di Ercole, alla modalità innica che Virgilio aveva utilizzato per Ercole e lui stesso per Teseo, accentuando piuttosto la modalità trenodica. Secondo la suggestione del grande monologo dell'eroe straziato dalla sofferenza nelle Trachinie di Sofocle (1046-1111), il poeta delle Metamorfosi 'trasferisce' il catalogo delle imprese nel luogo in cui avviene lo strazio del corpo possente di Ercole e la sua terribile fine, tra l'Eubea e il monte Eta. Nelle Trachinie l'eroe apostrofava le membra del suo corpo già possente per lamentare il tragico contrasto tra le gesta che esse avevano potuto compiere e l'attuale impotenza di fronte al dolore che lo annientava:

Soph. Trach. 1089-1104

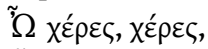

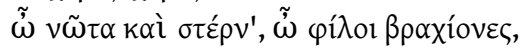

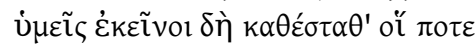

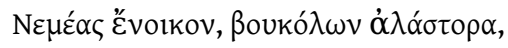

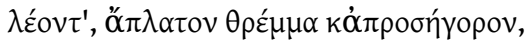

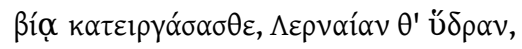

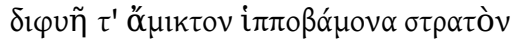

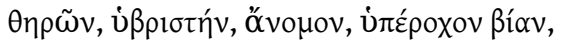

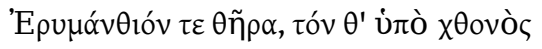

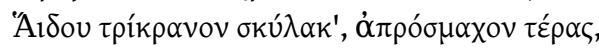

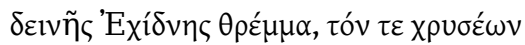

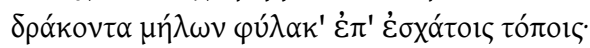

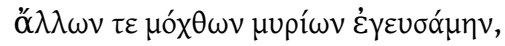

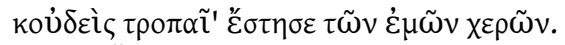

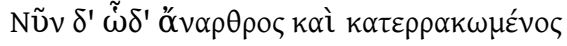

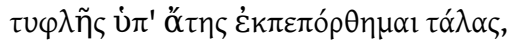

"Mie mani, e petto, e spalle, e braccia, foste voi a piegare con la vostra forza il leone

di Nemea, minaccia dei pastori, belva inaccostabile; e l'idra di Lerna, e la doppia

natura dei centauri, uomini e cavalli, feroci, senza legge, strapotenti; e il mostro di 
Erimanto e il cane a tre teste di Ade, invincibile figlio della spaventosa Echidna, e il drago custode delle mele d'oro, ai confini del mondo. E altre mille prove ho gustato; nessuno ha avuto vittoria contro il mio braccio. Ora, spezzato e distrutto, sono preda di una cieca sciagura" (trad. G. Paduano)

Nelle Metamorfosi, Ercole rivolge a se stesso e al suo corpo (tra Ich-Stil e apostrofe) una specie di lamento funebre che oppone all'accanimento della divinità persecutrice un ben più ampio catalogo di imprese (ne sono enumerate ben 16, rispetto alle 6 di Sofocle), che sembra mettere in discussione ogni possibile teodicea:

Ov. met. 9, 176-204

'cladibus,' exclamat 'Saturnia, pascere nostris: pascere, et hanc pestem specta, crudelis, ab alto, corque ferum satia. vel si miserandus et hosti, hoc est, si tibi sum, diris cruciatibus aegram invisamque animam natamque laboribus aufer. mors mihi munus erit; decet haec dare dona novercam. ergo ego foedantem peregrino templa cruore Busirin domui? saevoque alimenta parentis Antaeo eripui? nec me pastoris Hiberi forma triplex, nec forma triplex tua, Cerbere, movit? vosne, manus, validi pressistis cornua tauri? vestrum opus Elis habet, vestrum Stymphalides undae, Partheniumque nemus? vestra virtute relatus Thermodontiaco caelatus balteus auro, pomaque ab insomni concustodita dracone? nec mihi centauri potuere resistere, nec mi Arcadiae vastator aper? nec profuit hydrae crescere per damnum geminasque resumere vires? quid, cum Thracis equos humano sanguine pingues plenaque corporibus laceris praesepia vidi, visaque deieci, dominumque ipsosque peremi? his elisa iacet moles Nemeaea lacertis: hac caelum cervice tuli. defessa iubendo est saeva Iovis coniunx: ego sum indefessus agendo. sed nova pestis adest, cui nec virtute resisti nec telis armisque potest. pulmonibus errat ignis edax imis, perque omnes pascitur artus. at valet Eurystheus! et sunt, qui credere possint esse deos?'

“'Della sventura mia' esclama, 'o Saturnia, godi! Godi, e contempla, crudele, questa peste da lassù, sazia il tuo cuore feroce! Ma se anche come nemico ti faccio pietà, sottrai all'orrenda tortura una vita di sofferenze, insopportabile, nata solo per la fatica! La morte sarà per me un dono: un dono ben degno di una matrigna! Non fui io a domare Busiride che insozzava i templi col sangue straniero? a privare delle forze materne il crudele Anteo? a non tremare davanti al triplice aspetto del pastore d'Iberia, né al triplice tuo aspetto, o Cerbero? Non ho forse con voi, o mani, piegato le corna del toro possente? con voi compiuto le imprese dell'Elide, delle acque stinfaliche, dei boschi del Partenio? con voi conquistato la cintura scolpita nell'oro del Termodonte, con voi conquistato i pomi custoditi dal drago senza sonno? e poterono forse resistermi i Centauri, o il cinghiale che devastava l'Arcadia? e giovò forse all'Idra ricrescere a ogni ferita e recuperare due volte le forze? e quella volta che vidi i cavalli del Trace ingrassati di sangue umano e le greppie piene di corpi squartati, non le vidi, le distrussi e uccisi cavalli e padrone? Da queste mie braccia soffocato giace inerte l'enorme Nemeo, su queste mie spalle ho sorretto il cielo. Stancata si è a forza di dare ordini la sposa di Giove: io non ancora di eseguirli. Ma una nuova peste ora m'assale, cui non si resiste né col 
coraggio né con le armi: nei polmoni, dentro, si diffonde un fuoco divorante e di tutte le membra si pasce. Euristeo, invece, è vivo e vegeto! $\mathrm{E}$ c'è ancora chi crede nell'esistenza degli dèi?"' (trad. G. Chiarini) catalogo delle imprese di Ercole assume nell'epistola di Deianira, che, secondo la poetica delle Heroides, propone il punto di vista femminile rispetto al mondo dell'epica e della tragedia. E' anzitutto utile ricordare che, nell'ottica di Deianira, il complesso delle imprese di Ercole e quindi la forma del catalogo, lungi dal perdere importanza, si impone come struttura dominante dell'epistola. La finestra sul mito in cui Deianira scrive la sua lettera ha un ruolo determinante: l'eroina ha appena ricevuto la notizia dell'ulteriore gloriosa impresa guerresca del suo sposo (la presa di Ecalia), ma ha appreso anche di Iole, che minaccia il suo ruolo di sposa e amante: si è dunque risolta a ricorrere all'estrema risorsa del filtro d'amore che il centauro Nesso morente le aveva insidiosamente suggerito. La tunica, imbevuta del sangue di Nesso contaminato dal veleno dell'Idra, è stata consegnata, per suo incarico, al marito. La consapevolezza dell'effetto disastroso di quella tunica sopraggiungerà solo quando la lettera sta per essere conclusa:

Ov. her. 9, 143-4

scribenti nuntia venit

fama, virum tunicae tabe perire meae.

"Proprio mentre ti scrivo mi giunge la notizia che il mio sposo sta morendo per il

veleno della mia tunica." (trad. G. Rosati)

Nel confrontarsi con le imprese del marito, non sono dunque disponibili, per Deianira, né la modalità innico-celebrativa né quella trenodica, che ella non ha ancora motivo di prendere in considerazione. La notizia del successo dell'eroe come occasione di un canto di lode e di trionfo è invece una possibilità prospettata all'esordio, per essere immediatamente contraddetta e scartata:

Ov. her. 9, 1-2

Gratulor Oechaliam titulis accedere nostris;

victorem victae succubuisse queror.

"Mi compiaccio che Ecalia si aggiunga ai nostri titoli di gloria, ma che il vincitore

abbia ceduto alla vinta, lo deploro." (trad. G. Rosati)

Deianira si presenta come una donna che si identificherebbe ben volentieri col ruolo eroico del marito e sarebbe naturalmente incline a considerare anche propria ogni sua impresa, come un ulteriore titulus di gloria che si aggiunge alla serie che costituisce un patrimonio comune (titulis accedere nostris), Ciò è però reso impossibile dal fatto che,

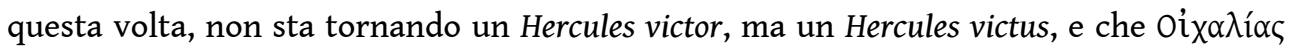

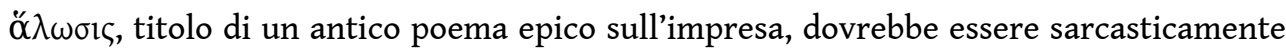

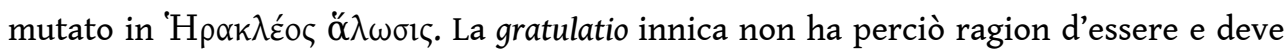
lasciare il posto alla querimonia elegiaca.

Nello spazio della querimonia, la series immensa laborum (v. 5) viene continuamente chiamata in causa, come singoli episodi e soprattutto come insieme, per denunciare lo scandalo dell'infedeltà di Ercole al suo ruolo, all'eroismo virile di cui invece proprio Deianira rivendica di essere l'unica autentica custode. Il comportamento recente di Ercole, ora con Iole, ma prima anche e soprattutto con Onfale, appone il coronamento di una turpis nota sui facta priora, che a questo punto hanno il paradossale risultato di conferire notorietà non alla sua gloria, ma alla sua vergogna. 
Il catalogo dei facta priora di Ercole gareggia in completezza, nell'epistola, con il lamento funebre a se stesso delle Metamorfosi: conta ben 15 occorrenze di cui 4 menzionate due volte, per un totale di 19:1) Presa di Ecalia: vv. 1-2; 2) Ercole si sostituisce ad Atlante: vv. 17-8; 3) Ercole bambino strozza i serpenti: 21-2; 4) pericolosi avversari di Ercole nelle preoccupazioni di Deianira: serpenti (Idra?), cinghiali (Erimanto), leoni (Nemeo), cani a tre teste (Cerbero): 37-8; 5) Atlante: v. 58; 6) Leone nemeo: 61-2; 7) Cavalle di Diomede: vv. 67-8; 8) Busiride: vv. 69-70; 9) Anteo: vv. 71-2; 10) Serpenti strozzati da Ercole bambino: vv. 85-6; 11) Cinghiale di Erimanto: vv. 87-8; 12) Cavalle di Diomede: vv. 89-90; 13) Gerione: vv. 91-2; 14) Cerbero: vv. 93-4; 15) Idra di Lerna: vv. 95-6; 16) Anteo: vv. 97-8 ; 17) Centauri: vv. 99-100; 18) Acheloo: vv. 139-40; 19) Nesso. vv. 141-2.

Spicca in particolare la serie dei vv. 85-100, in cui sette delle più note tra le fatiche (Cinghiale di Erimanto, Cavalle di Diomede, Gerione, Cerbero, Idra, Anteo, Centauri) fanno parte di un catalogo delle proprie imprese che Ercole, in abito femminile e ai piedi di Onfale, esibirebbe per compiacere e affascinare la regina. Deianira esercita tutta la sua ironia per l'incauto uso improprio della serie catalogica che Ercole faceva in quella situazione imbarazzante:

Ov. her. 9, 105-10

i nunc, tolle animos et fortia facta recense; quo tu non esses, iure vir illa fuit.

qua tanto minor es, quanto te, maxime rerum, quam quos vicisti, vincere maius erat.

illi procedit rerum mensura tuarumcede bonis; heres laudis amica tuae.

"Suvvia ora, esalta il tuo coraggio e passa in rassegna le tue gesta valorose. Non essendolo tu, a buon diritto l'uomo fu lei; le sei di tanto inferiore di quanto il vincere te, il più grande di tutti, era più che sconfiggere coloro che tu hai vinto. La misura delle tue gesta va a tutto vantaggio di lei; rinuncia al tuo patrimonio: la tua amica eredita la tua gloria." (trad. G. Rosati, con modifiche)

31 L'Ercole di Sofocle, come ha osservato Sergio Casali, piegato dalla sofferenza e costretto a poco virili lamenti, si vedeva 'femminilizzato' ad opera di Deianira ${ }^{22}$. La Deianira ovidiana rovescia preventivamente questa accusa sulle sue paelices e in particolare su Onfale. La rassegna dei fortia facta, eseguita in una situazione tanto imbarazzante, rende evidente che non ne può scaturire nessun orgoglio eroico come quello che si esprime nell'inno. La virilità di Ercole è ormai appannaggio di Onfale: "tu sei di tanto inferiore a lei di quanto vincere te, il più grande di tutti, era impresa più grande che vincere quelli che tu hai vinto". A questo appunto serve ora il catalogo: in un gioco puntiglioso di calcoli e di misurazioni, la misura delle gesta di Ercole va a tutto vantaggio di Onfale. I beni di Ercole (la gloria, l'onore) spettano, per proprietà transitiva, all'amica. L'invito a cedere bonis, più che un accenno all'istituto giuridico della cessio bonorum (come viene generalmente inteso) credo rimandi all'immagine diatribica dell'incalzare dell'erede ${ }^{23}$. Ironia, stavolta involontaria, per un personaggio che è effettivamente sul punto di morire. 


\section{BIBLIOGRAFIA}

Baldo 1995: G. Baldo, Dall'Eneide alle Metamorfosi. Il codice epico di Ovidio, Padova 1995.

Barchiesi 1989; A. Barchiesi, Voci e istanze narrative nelle Metamorfosi di Ovidio, “MD” 23, 1989, pp. 55-97.

Barchiesi 2001: A. Barchiesi, Speaking volumes. Narrative and intertext in Ovid and other Latin poets, London 2001.

Casali 1995: S. Casali, P. Ovidii Nasonis, Heroidum epistula IX. Deianira Herculi, Firenze 1995.

Citroni 1989: M. Citroni, Dedicatari e lettori della poesia elegiaca, in G. Catanzaro, F. Santucci (a cura di), Tredici secoli di elegia latina, Assisi 1989, pp. 93-143.

Citroni 1995: M. Citroni, Poesia e lettori in Roma antica, Roma-Bari 1995.

Delvigo 2020: M. L. Delvigo, Verso Tomi: il poeta epico ritrova l'elegia, in M. Möller (Hrsg.), Excessive writing. Ovids Exildichtung, Heidelberg 2020, pp. 39-55.

Feeney 1986: D. C. Feeney, Epic Hero and Epic Fable, “Comparative Literature” 38, 1986, pp. 137-158.

Galinsky 1966; G. K. Galinsky, The Hercules-Cacus Episode in Aeneid VIII, “AJPh” 87, 1966, pp. 1851.

Galinsky 1972; G. K. Galinsky The Herakles Theme: The Adaptations of the Hero in Literature from Homer to the Twentieth Century, Oxford 1972.

Galinsky 1972a: G. K. Galinsky, Hercules Ovidianus (Metamorphoses 9, 1-272), “Wien. St.” 85, 1972, pp. 93-115.

Galinsky 1975: G. K. Galinsky, Ovid's Metamorphoses. An Introduction to the Basic Aspects, Oxford 1995.

Giuseppetti 2015: M. Giuseppetti, Bacchilide. Odi e frammenti, Milano 2015.

Kenney 2011: Ovidio, Metamorfosi, Volume IV, Libri VII-IX. A cura di E. J. Kenney, Milano 2011

Labate 2010: M. Labate, Passato remoto. Età mitiche e identità augustea in Ovidio, Pisa-Roma 2010.

Liapis 2006: V. Liapis, Intertextuality as irony: Heracles in epic and in Sophocles, "G\&R" 53, 2006, pp. 48-59.

Lipka 2018, M. Lipka, Aretalogical Poetry: A Forgotten Genre of Greek Literature .Heracleids and Theseids, "Philologus" 162, 2018, pp. 208-31.

Mirto 1997: M. S. Mirto, Euripide, Eracle, Milano 1997.

Möller 2020: M. Möller, Ovid und Odysseus. Zur Rhetorik des Exils, in Ead. (Hrsg.), Excessive writing. Ovids Exildichtung, Heidelberg 2020, pp. 57-77.

Swift 2011; L. A. Swift, Epinician and tragic worlds: the case of Sophocles' Trachiniae, in L. Athanassaki, E. Bowie (eds), Archaic and Classical Choral Song: Performance, Politics and Dissemination, Berlin-Boston 2011, pp. 391-413. 
Tandoi 1992: V. Tandoi, Albinovano Pedone e la retorica giulio-claudia delle conquiste, in Scritti di filologia e di storia della cultura classica, Pisa 1992, 509-85 (saggio apparso originariamente in "SIFC" 36, 1964, pp. 129-168 e 39, 1967, pp. 5-66).

Tsagalis 2011: Ch. Tsagalis, The Heracles theme: from inscriptional to literary epigram, "RFIC" 139, 2011, pp. 43-100.

\section{NOTE}

1. Riprendo qui in breve la trattazione di Labate 2010, pp. 19-21, 39-40, 83-93.

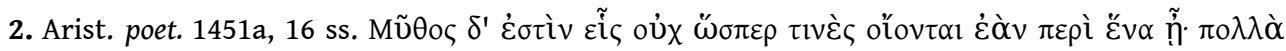

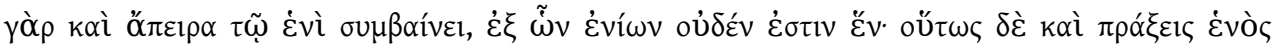

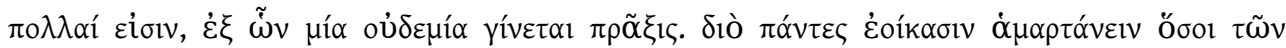

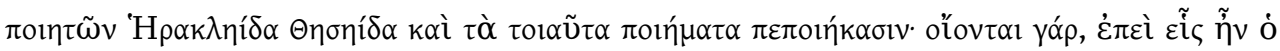

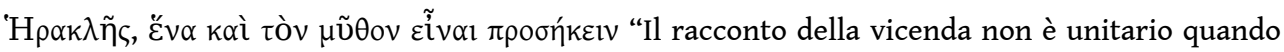
riguarda una sola persona, come credono alcuni: difatti possono concorrere insieme molti e interminabili accadimenti, senza che dai singoli si ricavi unità. Così anche le azioni di un singolo sono molte, ma non ne risulta per niente un'azione unitaria. Quindi sono evidentemente in errore tutti quei poeti che hanno composto una Eracleide, una Teseide, e simili poemi: siccome Eracle è uno solo, credono che uno sia di conseguenza anche il racconto" (trad. C. Gallavotti). Un'ottima trattazione della questione del ruolo dell'eroe nell'epica antica alla luce delle riflessioni di Aristotele in Feeney 1986 (sono grato della segnalazione all'anonimo referee della rivista).

3. La trattazione fondamentale di questa complessa materia resta Galinsky 1972. Da vedere anche Liapis 2006.

4. Vedo ora che, da una prospettiva in qualche misura affine alla mia, questo aspetto è messo a fuoco da Lipka 2018.

5. Per la raffinata concentrazione formale della sintesi come munus cortese alla coppia di amici, vedi Citroni 1989, p. 127 (= Citroni 1995, pp. 406-7).

6. Su Ulisse come ipotesto epico dell'Ovidio dell'esilio vedi Möller 2020 e Delvigo 2020, p. 44, n. 15 con bibliografia.

7. Tra i lavori più significativi, ricordo solo Galinsky 1975, pp. 217-51 e Baldo 1995, pp. 29-109, con ampia bibliografia.

8. Da vedere Galinsky 1972a e 1972, pp. 157-8. Vedi anche Labate 2010, pp. 87-88.

9. Che nello scontro con Ercole il dio fluviale assuma varie forme sembra essere innovazione ovidiana: cfr. Kenney 2011, p. 395.

10. Ovidio sembra più interessato a questi giochi ironici, che a dare un quadro coerente e privo di contraddizioni della cronologia relativa delle fatiche di Ercole: cfr. Kenney 2011, p. 396.

11. La rappresentazione in questi termini dello scontro tra Ercole e Acheloo, prima che in Ovidio, era importante nel primo stasimo delle Trachinie di Sofocle (vv. 497-530): cfr. Swift 2011, pp. 393-6.

12. Sul ruolo di Teseo come narratario cfr. Barchiesi 1989, pp. 57 ss. (= Barchiesi 2001, pp. 50 ss.).

13. Call. Hec. 68-69 Hollis (268-9 Pf.).

14. Cfr. Labate 2010, pp. 45-50, con essenziali indicazioni bibliografiche,

15. Cfr. Labate 2010, pp. 85-6.

16. Verg. Aen. 8, 219-20 hic vero Alcidae furiis exarserat atro / felle dolor; Verg. Aen. 8, 228-30 ecce furens animis aderat Tirynthius omnemque / accessum lustrans huc ora ferebat et illuc, / dentibus infrendens.

17. Vedi ora Lipka 2018. 
18. Vedi Giuseppetti 2015, pp. 88-91, con indicazione della bibliografia rilevante.

19. Cfr. Tandoi 1992, pp. 509-10. Negli stessi anni Caro, precettore dei figli di Germanico, attendeva a una Eracleide, confermando il revival di queste figure eroiche in un contesto culturale interessato ad ambizioni ecumeniche di conquista (Ov. Ex P. 4, 13, 11-2; 4, 16, 7).

20. Cfr. Kenney 2011, p. 270.

21. Un'ottima analisi in Mirto 1997, pp. 136-7.

22. Casali 1995, pp. 11-17.

23. Hor. carm. 2, 3, 17-20 cedes coemptis saltibus et domo / villaque flavos quam Tiberis lavit, / cedes et exstructis in altum / divitiis potietur heres.

\section{RIASSUNTI}

L'articolo studia le modalità secondo cui in Virgilio e soprattutto in Ovidio sono trattate le storie relative agli 'eroi più grandi' (Ercole e Teseo). L'epica augustea sceglie la strada della narrazione ampia di singoli episodi in cui si dispiegano le qualità eroiche del personaggio (Ercole contro Caco in Virgilio, Ercole contro Acheloo, Ercole contro Nesso in Ovidio), ma al tempo stesso non rinuncia alla possibilità, anzi quasi alla necessità, di un riferimento trasversale ad altre storie della serie e anzi alla serie nel suo complesso. La struttura formale attraverso cui questo riferimento si attua è quella di catalogo delle imprese, che rielabora moduli innici e aretalogici desunti dalla tradizione della lirica e della tragedia, sia nella forma del canto di celebrazione della vittoria (epinicio), sia in quella della celebrazione delle virtù e delle imprese dell'eroe morto (threnos).

\section{INDICE}

Parole chiave : Ercole, Teseo, Virgilio, Ovidio, Catalogo, Aretalogia 